\title{
PROPOSTA DE RELANÇAMENTO PARA O RESTAURANTE DE COMIDA JAPONESA SUSHIAKI
}

\author{
Dionathan Bittencourt Cabral Vilela ${ }^{1}$ \\ Gabriele Machado ${ }^{2}$ \\ Ísis Figueiredo Araújo ${ }^{3}$ \\ Maria Clara Gíglio Felipe ${ }^{4}$ \\ Maria Luíza Gíglio Felipe ${ }^{5}$ \\ Ana Carolina Marini Figueira dos Santos ${ }^{6}$
}

Resumo: O tema do trabalho é o relançamento do restaurante de comida japonesa Sushiaki. Tem-se como objetivo o estudo de novas estratégias de marketing para maior sucesso da empresa. O trabalho utilizou pesquisas de mercado realizadas com o público em geral, com a finalidade de saber sua opinião sobre a proposta de relançamento. Foi realizada a pesquisa pelo método quantitativo, além da realização da análise de SWOT, com o objetivo de analisar fatores que afetam a empresa. No decorrer do trabalho foi realizado um briefing com o dono da empresa para recolher informações relacionadas aos seus desejos quanto ao relançamento de seu restaurante delivery. Com o relançamento do restaurante a empresa poderá atrair e fidelizar clientes através de desenvolvimento de identidades visuais, participações em eventos e divulgação virtual.

Palavras-chave: Restaurante; Relançamento; Comida Japonesa.

\footnotetext{
1 Univap/Colégio Técnico “Antônio Teixeira Fernandes”, Brasil. E-mail: isisfgrd.cats@gmail.com.

2 Univap/Colégio Técnico “Antônio Teixeira Fernandes”, Brasil. E-mail: dionavilela@gmail.com.

3 Univap/Colégio Técnico “Antônio Teixeira Fernandes”, Brasil. E-mail: gabrielemachado14@gmail.com.

4 Univap/Colégio Técnico “Antônio Teixeira Fernandes”, Brasil. E-mail: mariaclaragigliofelipe@gmail.com.

5 Univap/Colégio Técnico “Antônio Teixeira Fernandes”, Brasil. E-mail: marialuizagigliofelipe1@gmail.com.

6 Univap/Colégio Técnico “Antônio Teixeira Fernandes”, Brasil. E-mail: mariniturismo@gmail.com.
} 